\title{
LA DIMENSIONE CONTEMPLATIVA DELLA LITURGIA
}

\author{
THE CONTEMPLATIVE DIMENSION OF THE LITURGY
}

A b s t r a c t. The relationship between liturgy and contemplation is fundamental to Christian living. Of its very nature, contemplation is concerns the mystery, because its object is the mysteries of salvation proclaimed in the Word of God, fulfilled in the person of Christ, and celebrated in the liturgy. Authentic contemplation has to be nourished by the Word proclaimed and by the celebration of the mysteries of salvation. Moreover, all liturgy is ordered towards contemplation as to its proper perfection - not so as to stop being liturgy, but to become contemplative liturgy. Our article seeks to highlight the inseparable bond between these two components of Christian existence, a bond guarantees both the authenticity of contemplation and the fruitfulness of participation in the liturgical celebration.

Keywords: Liturgy; contemplation; baptism; mystery, celebration; Church.

Nell'intricata storia del rapporto tra liturgia e spiritualità, in genere, e liturgia e contemplazione, in modo particolare, $i$ due termini sono stati per lungo tempo considerati opposti, almeno nell'ambito della Chiesa occidentale, come se una escludesse l'altra. Storicamente, il distacco tra liturgia e spiritualità ha generato un impoverimento della contemplazione e della vita contemplativa nella Chiesa. La vita contemplativa si è allontanata dalle sorgenti autentiche che sono le realtà della fede, proclamate dalla Parola di Dio e comunicate nella vita sacramentale liturgica. La liturgia è stata considerata, per

VALÉRY BITAR OCD - Carmelitano scalzo, nato in Libano, ha conseguito il dottorato in liturgia presso il Pontificio Ateneo sant'Anselmo - Roma. Attualmente è docente di Liturgia, di Spiritualità liturgica e di Spiritualità patristica alla Pontificia Facoltà Teologica - Pontificio Istituto di Spiritualità Teresianum (Roma); e-mail: bitar@teresianum.net 
lunghi secoli, un insieme complesso di riti, rubriche e cerimonie. Ovviamente, oggi quest'opposizione è ormai superata, mentre rimane necessario un continuo approfondimento dell'argomento, per riscoprire il ricco legame che intercorre tra la leitourgia, prima scuola di spiritualità, e la contemplazione alla quale ogni battezzato è chiamato e abilitato. Il nostro articolo cercherà di mettere in luce l'inscindibile rapporto tra questi due aspetti fondamentali della vita cristiana, inserendosi nel solco di una ricerca continua di un'adeguata interazione tra di loro. Rimane sempre attuale la necessità di una liturgia vissuta in stile contemplativo e una contemplazione che sia sempre più radicata nella celebrazione dei misteri della fede ${ }^{1}$.

Studiando l'argomento in modo approfondito, ci accorgiamo che il rapporto tra liturgia e contemplazione non è una questione secondaria. Vagaggini, grande studioso della teologia della liturgia, la considera «una questione centrale [...] addirittura questione basilare di vita cristiana» ${ }^{2}$. Špidlík, dal canto suo, ritiene che «il legame fra celebrazione liturgica e contemplazione cristiana va ben al di là di un riferimento ridotto semplicemente all'esemplarità. La conformazione a Cristo, realizzata con l'ingresso nella comunione ecclesiale, ha creato la possibilità stessa di un cammino „spirituale” cristiano» ${ }^{3}$. Un erudito come Daniélou, attento al rapporto tra vita liturgica e vita mistica, afferma che tutta la cultura cristiana consiste nel cogliere i rapporti esistenti tra la Bibbia e la Liturgia, tra il Vangelo e l'Escatologia, tra la Mistica e la Liturgia ${ }^{4}$.

Riteniamo importante notare che l'abilitazione alla contemplazione è fondata nel battesimo, chiamato sacramento dell'illuminazione, che apre la porta all'esperienza dei misteri creduti e celebrati. Partendo da questa verità di fede, è facile notare che ciò che viene spesso trascurato è la coscienza del fatto che l'abilità a una vita contemplativa è possibile a tutti e non è l'appannaggio di alcuni specialisti. Nell'esperienza cristiana, la contemplazione consiste in un'unificazione interiore attorno ad un oggetto della nostra fede, a un mistero interiorizzato e pregato ${ }^{5}$. San Paolo Sesto, nella sua omelia del 7 di-

${ }^{1}$ Cf. Jesús Castellano, Liturgia y vida espiritual. Teología, celebración, experiencia (Barcelona: Centre de Pastoral Litúrgica, 2006), 273.

${ }^{2}$ Cipriano Vagaggini, "Contemplazione nella Liturgia e Contemplazione fuori della Liturgia," Rivista di ascetica e mistica 7(Gennaio-Febbraio 1962), 1: 8-9.

3 Tomáš Špidlík e Innocenzo Gargano, La spiritualità dei Padri greci e orientali (Roma: Borla, 1983), 167-68.

${ }^{4}$ Cf. Jean Daniélou, "Le symbolisme des rites baptismaux," Dieu Vivant 1(1945): 17.

${ }^{5}$ Cf. Castellano, Liturgia y vida espiritual, 273. 
cembre 1965, pronunciata a conclusione del Concilio Vaticano II, afferma: "Quello sforzo di fissare in lui [Dio] lo sguardo e il cuore, che diciamo contemplazione, diventa l'atto più alto e più pieno dello spirito, l'atto che ancor oggi può e deve gerarchizzare l'immensa piramide dell'attività umana» ${ }^{6}$.

\section{ALCUNE ANNOTAZIONI BASILARI}

La contemplazione, per sua natura, possiede un aspetto „misterico”, perché ha come oggetto il mistero salvifico di Dio rivelato in Cristo che culmina nel suo mistero pasquale, celebrato e attualizzato nella liturgia della Chiesa. A livello personale, la contemplazione è un atto di unificazione della mente, del cuore, della fede e dell' amore. Ovviamente, la questione della contemplazione non si esaurisce in una tecnica: è lo Spirito Santo "effuso nei nostri cuori" che attua in noi questo slancio d'amore e ci unisce a Dio.

Partendo da questa considerazione, affermiamo l'importanza, da parte di quella contemplazione che vuole definirsi autentica, di nutrirsi della liturgia, della Parola proclamata e della celebrazione dei misteri della Storia della Salvezza. In altri termini, deve abbeverarsi alla fonte della Parola, della preghiera, dei sacramenti e della ricchezza dei testi liturgici. È un dato di fatto che le verità della fede, proclamate nella Parola di Dio, sono comunicate nella liturgia sacramentale. L'impegno dei Padri della Chiesa si prefiggeva sempre l'obiettivo di illustrare questa verità mettendola al servizio di un autentico impegno cristiano. Daniélou, analizzando il pensiero di Gregorio di Nissa, mostra che la Theorìa per il Nisseno è la contemplazione del Mistero nella sua sostanza atemporale. Per mezzo di quest'analisi ci accostiamo alla liturgia che è, in modo specifico, la ripresentazio$\mathrm{ne}^{7}$, nel tempo, del Mistero. Da qui, Daniélou sostiene che la Theorìa liturgica, la Theoria scritturistica e la Theorìa mistica sono fondamentalmente gli aspetti diversi di un'unica realtà ${ }^{8}$.

\footnotetext{
${ }^{6}$ Paolo VI, “Omelia nella 9 a sessione pubblica. 7 dicembre 1965," Enchiridion Vaticanum 1(1962-1965): 297.

${ }^{7}$ Il termine latino Repraesentaretur, usato dal concilio di Trento, può essere tradotto in italiano con due termini "rappresentare" e "ripresentare". La parola "rappresentazione" potrebbe farci cadere in un equivoco (mimetico-teatrale) rispetto all'evento del quale si fa memoria. Il termine "ripresentazione" invece esprime il rendere ancora presente, riattualizzare un evento che non appartiene più all'uomo, ma all'eternità di Dio e si rende presente nel "tempo celebrativo" o "tempo liturgico". Cf. Pietro Angelo Muroni, Il Mistero di Cristo nel tempo e nello spazio. La celebrazione cristiana (Roma: Urbaniana University Press, 2014), 40-41.

${ }^{8}$ Cf. Jean Daniélou, Platonisme et théologie mystique. Essai sur la doctrine spirituelle de Saint Grégoire de Nysse (Paris: Éditions Montaigne, 1954), 172-73.
} 
In epoca recente, e con un tono polemico, i coniugi Maritain, in un celebre articolo sul rapporto tra liturgia e contemplazione, consideravano che la liturgia richiede che l'anima tenda alla contemplazione, e la partecipazione alla vita liturgica sia una preparazione all'unione a Dio per una contemplazione d'amore ${ }^{9}$. Nonostante quest'affermazione, i Maritain ritenevano che la contemplazione, come frutto delle virtù teologali di fede, speranza e carità, e attuazione dei doni dello Spirito Santo, fosse un fine superiore al culto liturgico, appartenente alle virtù della religione, parte potenziale della giustizia e quindi riducibile a una virtù morale. Per loro sarebbe erroneo considerare che la semplice partecipazione alla liturgia porti la nostra vita spirituale ad un grado superiore rispetto a ciò che conduce all'unione con Dio nella contemplazione $^{10}$. Il "culto liturgico" è in se stesso un fine, ma che tende per natura a preparare e ad avviare quelli che vi partecipano ad un fine più elevato che è la contemplazione. La partecipazione alla vita liturgica prepara le anime al raccoglimento sopranaturale e all'unione contemplativa; però, allo stesso tempo, la liturgia non è né la via unica né la via indispensabile per la contemplazione ${ }^{11}$.

L'errore dei coniugi Maritain consisteva nell'accentuare una separazione tra liturgia e contemplazione, nel porre quest'ultima a un livello superiore rispetto alla prima, e nel considerare il culto liturgico ordinato alla contemplazione. Vagaggini, rispondendo alle tesi dei Maritain, mostra che «il culto liturgico è ordinato alla contemplazione intraliturgica come alla massima perfezione interiore della partecipazione, nello stesso tempo normalmente attiva e comunitaria, all'azione liturgica» ${ }^{12}$. L'autore esce dalla dicotomia Liturgia/contemplazione e considera quest'ultima come l'aspetto interno della liturgia arrivato a perfezione. In questo senso, la contemplazione è intrinseca all'atto liturgico, mentre la contemplazione che si compie fuori della liturgia è ordinata alla liturgia ed è frutto del culto liturgico. Come risultato, «si deve dire che il culto tende ed è ordinato intrinsecamente alla contemplazione come alla perfezione interna di se stesso, non già per cessare di essere culto, ma per diventare culto contemplativo o contemplazione cultica» ${ }^{13}$.

\footnotetext{
${ }^{9}$ Cf. Jacques Maritain et Raïssa Maritain, Liturgie et contemplation (Bruges: Desclée de Brouwer, 1959), 13.

${ }^{10} \mathrm{Cf}$. Maritain et Maritain, Liturgie et contemplation, 28-29.

${ }^{11}$ Cf. Maritain et Maritain, Liturgie et contemplation, 79.94-95.

12 Vagaggini, "Contemplazione nella Liturgia," 12.

13 Vagaggini, "Contemplazione nella Liturgia," 14-15.
} 


\section{LA RADICE BATTESIMALE E LA COMUNIONE ECCLESIALE}

Leggendo attentamente i Padri della Chiesa, e in modo particolare i Padri orientali, è facile notare quanto era naturale per loro collegare la contemplazione e l'esperienza contemplativa nel suo insieme, alla celebrazione liturgica. Questo fatto trova la sua radice nel battesimo, dove l'impronta battesimale ci fa entrare nella vita dello Spirito manifestata nella Chiesa, la carne di Cristo: «A tale vita e alla incorruttibilità questa carne può partecipare se ad essa si unisce lo Spirito Santo. Nessuno può esprimere e dire quello che il Signore ha preparato per i suoi eletti» ${ }^{14}$. Da sempre, la liturgia ha riconosciuto ai battezzati la pienezza della dignità cristiana espressa nella dimensione ecclesiale: «L'uomo liturgico è colui che vive questa dinamica dello Spirito: è afferrato in tutto il suo essere dal mistero celebrato [...] attento alla voce dello Spirito, il credente vive il mistero liturgico inserendosi nel cammino che la Chiesa compie con il suo Signore» ${ }^{15}$.

Con il battesimo si realizza l'inserimento nella Chiesa, nella comunità radunata, l'assemblea, luogo ideale della preghiera cristiana. Origene afferma: «Il miglior posto per pregare è proprio quello, dove i santi si radunano in assemblea» ${ }^{16}$. Perché nella Chiesa, corpo di Cristo, è Cristo il capo che prega il Padre e «associa sempre a sé la Chiesa, sua sposa amatissima» $(\mathrm{SC} 7)^{17}$. In questo modo, l'orante «parteciperà alla preghiera del Verbo di Dio, il quale è anche in mezzo a coloro che lo ignorano e non diserta le preghiere di nessuno. Il Figlio di Dio infatti è il sommo sacerdote delle nostre offerte e l'avvocato presso il Padre. Prega per quelli che pregano, intercede per quelli che intercedono» ${ }^{18}$. Per questo motivo, la preghiera liturgica può essere considerata, a giusto titolo: «Culmine, norma, criterio, punto di riferimento, sorgente, sacramento di ogni preghiera cristiana, non in senso meramente giuridico-istituzionale ma oggettivo-contenutistico. Oggettivamen-

${ }^{14}$ Pseudo-Clemente, "Omelia dello Pseudo-Clemente", in I Padri apostolici, ed. e trad. Antonio Quacquarelli (Roma: Città Nuova Editrice, 1981³), 230.

15 Bonifacio Baroffio, "Liturgia ed esperienza di Dio. Riflessioni liturgico-pastorali," in Liturgia Soglia dell'esperienza di Dio?, ed. Aldo Natale Terrin (Padova: Edizioni Messaggero Padova, 1982), 20.25. Il corsivo è dell'autore.

${ }^{16}$ Origene, La preghiera, XXXI, 6, trad. Giuseppe Del Ton (Roma: Arnoldo Mondadori Editore, 1984), 170.

${ }^{17}$ Costituzione conciliare sulla Liturgia: Sacrosanctum Concilium. D'ora in poi SC.

${ }^{18}$ Origene, La preghiera, X,2, 62. 
te, dato il suo carattere normativo, il contenuto della preghiera liturgica si accorda perfettamente con l'ideale della preghiera cristiana» ${ }^{19}$.

In ogni liturgia ecclesiale c'è un chiaro riferimento alla liturgia del cielo, a questo compimento di cui parla il libro dell'Apocalisse. Il Corpus dionisia$\mathrm{co}^{20}$ manifesta chiaramente questo legame e questa dinamica liturgica-ecclesiale che sfocia in una contemplazione dei "cieli nuovi" e della "terra nuova", pregustati nella liturgia della terra. In questa dinamica, Schmemann considera che «l'intera liturgia è sacramentale», cioè «un solo atto trasformante e un solo movimento d'ascensione», perché la liturgia della Chiesa «è sempre una anaphorá, una elevazione, un'ascensione» ${ }^{21}$. Questa elevazione si compie nella Chiesa e nella sua liturgia, preparando e pregustando la condizione dell'uomo rinnovato. In questa direzione vanno le parole di Gregorio di Nissa, nel suo commento sul Cantico dei cantici, considerando che nella Chiesa:

È creato un cielo nuovo, che è il firmamento della fede in Cristo [...] e viene preparata una terra nuova, la quale beve la pioggia che cade su di lei, e viene plasmato un altro uomo, che si rinnova per mezzo della generazione dall'alto a immagine di colui che lo ha creato [...] colui che guarda questo nuovo mondo della creazione secondo la Chiesa vede in esso colui che è ed è divenuto tutto in tutti ${ }^{22}$.

Quando il Nisseno contempla il "mistero della pietà" di cui parla san Paolo in 1Tim 3,16, vede l'unità di Cristo e della Chiesa: «Dunque, colui che guarda la Chiesa guarda direttamente Cristo, che si edifica e si accresce per mezzo dell'aggiunta di coloro che si salvano» ${ }^{23}$. In questo cammino, come Cristo guida verso la gloria del Padre, la Chiesa è la guida sicura nella via spirituale, e la sua liturgia luogo privilegiato per un'esperienza viva di Cristo. Infatti, «allo stesso modo che la potenza della Chiesa risiede nella sua vita

${ }^{19}$ Matias Augé, Spiritualità liturgica. «Offrite i vostri corpi come sacrificio vivente, santo e gradito a Dio» (Cinisello Balsamo: San Paolo, 1998), 100-101.

${ }^{20}$ L'identità di Dionigi l'Areopagita rimane un grande enigma. La collezione degli scritti attribuita a Dionigi, discepolo di san Paolo ad Atene (At 17,34), fu assai influente in tutte e tre le tradizioni cristiane, la latina, la greca e la siriaca. Il vero autore scriveva alla fine del V secolo, o all'inizio del VI.

${ }^{21}$ Alexander Schmemann, Per la vita del mondo. Il mondo come sacramento, trad. Maria Campatelli (Roma: Lipa, 2012), 57.

${ }^{22}$ Gregorio di Nissa, Omelie sul Cantico dei cantici, XIII, trad. Claudio Moreschini (Roma: Città Nuova Editrice, 1988), 297-98.

${ }^{23}$ Gregorio di Nissa, Omelie sul Cantico dei cantici, XIII, 295. 
attiva, la sua bellezza sta nella sua vita contemplativa. Questa non è soltanto una struttura funzionale per se stessa, bensì anche realtà, piena di senso, la quale diventa arte. Tale essa è quando prega: nella liturgia» ${ }^{24}$.

\section{LA LITURGIA SCUOLA DI CONTEMPLAZIONE}

Senza dubbio, la liturgia è il momento più privilegiato per entrare in una contemplazione autentica: «Esiste una preghiera contemplativa liturgica, che è preghiera contemplativa della Chiesa a titolo speciale, con dignità ed efficacia trascendenti la preghiera contemplativa puramente privata» ${ }^{25}$. Se quest'aspetto ci sfugge è perché manca la consapevolezza di vivere la liturgia come momento forte di attualizzazione del Mistero, oggetto di ogni contemplazione, e di parteciparne con un'intensa vita teologale. La liturgia è la celebrazione del Dio vivente, il Dio presente, dell'azione santificatrice del Kyrios, del Signore risorto, della sinergia con lo Spirito Santo. La verità del mistero di Cristo, attualizzato nella Liturgia, fonda la preghiera e la contemplazione autentica perché: «Buona è solo quella preghiera che viene dalla verità $[\ldots]$ la verità rende potente la preghiera ${ }^{26}$.

Quando, nelle nostre liturgie, viene meno il senso della presenza trinitaria, del Mistero celebrato, viene meno lo slancio contemplativo che appartiene alla natura stessa della liturgia ed è richiesto come una condizione fondamentale per partecipare attivamente alla liturgia: «Partecipare al mistero non è, quindi, un accessorio o un accidente del sacramento, ma la sua sostanza» ${ }^{27}$. Vagaggini considera che «quando l'unione interiore, che deve realizzarsi in ogni vera partecipazione attiva alla liturgia, arriva alla sua perfezione, essa diventa nello stesso tempo contemplazione o partecipazione contemplativa ${ }^{28}$. In questo senso, si deve dire che «non si può dare partecipazione piena e perfetta alla liturgia se non è nello stesso tempo partecipazione con-

\footnotetext{
${ }^{24}$ Romano Guardini, Lo spirito della liturgia. I santi segni, trad. Mario Bendiscioli (Brescia: Morcelliana, $2007^{11}$ ), 86 .

${ }^{25}$ Vagaggini, "Contemplazione nella Liturgia," 17.

${ }^{26}$ Guardini, Lo spirito della liturgia, 21.

27 Andrea Grillo, Eucaristia. Azione rituale, forme storiche, essenza sistematica (Brescia: Queriniana, 2019), 381. 690.

${ }^{28}$ Cipriano Vagaggini, Il senso teologico della liturgia (Roma: Edizioni Paoline, 1965 ${ }^{4}$ ),
} 
templativa. Ecco in che senso la liturgia è ordinata alla mistica come a un aspetto essenziale di se stessa» ${ }^{29}$.

Nella dinamica della liturgia, parole, preghiere, gesti e silenzi si alternano per formare un clima che apre all'esperienza viva del Mistero. Rimane necessario ricordare che l'oggetto delle nostre preghiere va al di là delle parole. Lo Spirito parla a noi a un livello più profondo, lì dove nessuna parola può raggiungerci, dove solo nella calma e nel silenzio riusciamo ad ascoltare. Una liturgia contemplativa ha bisogno di questo silenzio interiore e di pause di silenzio esteriori. La partecipazione attiva è al servizio di una liturgia contemplativa quando mettiamo il cuore a disposizione della presenza divina, un cuore attento, ricettivo, pacificato, pronto a gustare l'ineffabile dolcezza della presenza divina. L'intensità del vissuto liturgico ha bisogno del silenzio, dove nella tranquillità sentiamo il passaggio di Dio nel «sussurro di una brezza leggera» $(1 \operatorname{Re} 19,12)^{30}$. Nella liturgia, infatti, bisogna percorrere un itinerario interiore verso la Trascendenza; ma «rimane vero che la spiritualità liturgica è più ex-statica che in-statica, proclama il primato dell'oggetto [Dio] nel cui ambito i due poli - il soggetto e l'oggetto stesso - si armonizzano» ${ }^{31}$.

Tutta la fede cristiana ha un unico oggetto: il mistero di Cristo morto e risorto. Questo Mistero sussiste sotto modi diversi: è prefigurato nell'Antico Testamento; è compiuto storicamente nella vita terrestre di Cristo; è contenuto in mistero nei sacramenti; è vissuto misticamente nelle anime; si realizza socialmente nella Chiesa; si compie escatologicamente nel regno celeste ${ }^{32}$. La liturgia per sua natura sviluppa in noi un atteggiamento tipicamente contemplativo a diversi livelli, in un passaggio dal visibile all'invisibile ${ }^{33}$. Questo passaggio si compie in uno slancio di amore, di fede e di speranza: «Fede, speranza e carità coinvolgono tutta la vita, dalle radici alla fioritura, dalla semina alla raccolta dei frutti, fino all'eschaton/telos» ${ }^{34}$. La liturgia conduce

${ }^{29}$ Vagaggini, Il senso teologico della liturgia, 691.

${ }^{30}$ Cf. Robert Duggan, "Liturgical spirituality and liturgical reform," Spiritual Life 27 (Spring 1981), 1: 52-53.

${ }^{31}$ Mariano Magrassi, "Spiritualità liturgica. Riflessione pastorale," in Liturgia e spiritualità, ed. Secondo Mazzarello (Torino: Marietti, 1981), 56.

32 Cf. Daniélou, "Le symbolisme des rites baptismaux," 17.

${ }^{33}$ Cf. Castellano, Liturgia y vida espiritual, 277.

${ }^{34}$ Stefano Rosso, Un popolo di sacerdoti. Introduzione alla liturgia (Leumann: Elledici, $\left.2007^{2}\right), 460$. 
l'orante dal silenzio e dal gesto alla parola, poi dalla parola e dal gesto al silenzio della visione della fede ${ }^{35}$.

Su questo slancio ex-statico, caratterizzante della liturgia, Guardini osserva:

Nella liturgia l'uomo non guarda a sé, bensì a Dio; verso di Lui è diretto lo sguardo. In essa l'uomo non deve tanto educarsi, quanto contemplare la gloria di Dio. Il senso della liturgia è pertanto questo: che l'anima stia dinanzi a Dio, si effonda dinanzi a Lui, si inserisca nella Sua vita, nel mondo santo delle realtà, verità, misteri, segni divini, e così si assicuri la vera e reale vita sua propria ${ }^{36}$.

La contemplazione intraliturgica, ossia la liturgia contemplativa, non è altro che una «intelligenza fondata nell'epicentro spirituale della persona umana», ed è «un'intelligenza aperta all'azione dello Spirito Santo, e dunque si realizza nell'agape e anche agisce per mezzo della comunione». Per questo è un'intelligenza "contemplativa", che, «mentre scopre ciò che conosce, instaura anche un rapporto di comunione con quanto conosce» ${ }^{37}$. In questo modo, la liturgia diventa «un capire, è un cammino, è una realizzazione della propria umanità» ${ }^{38}$. Infatti, lo Spirito Santo guida quest'esperienza, essendo il «pedagogo della nostra preghiera così come è il mistagogo delle nostre celebrazioni» ${ }^{39}$. Egli guida a una piena comunione con il mistero celebrato e a una piena comunione con la comunità che celebra. Una liturgia contemplativa significa una celebrazione liturgica "integrale", per usare l'espressione di Corbon, che porta nel centro della fede e si riverbera in comunione ${ }^{40}$. Tutta quest'analisi abbraccia la riflessione di Gregorio di Nissa, dove la vita sacramentale è concepita come una "mistagogia", come un'iniziazione progressiva che conduce l'anima fino alle vette della vita mistica, fino alla "sobria ebbrezza"41. La mistagogia consiste nel leggere nei riti, il mistero di Cristo e contemplare, sotto i simboli, la realtà invisibile. Infatti, il sacramento secondo il Nisseno, e in modo particolare il battesimo, chiamato il

\footnotetext{
${ }^{35}$ Paul Grammont, "Liturgie et contemplation," in Liturgie et vie spirituelle (Paris: Éditions Beauchesne, 1977), 124.

${ }^{36}$ Guardini, Lo spirito della liturgia, 76.

${ }^{37}$ Marco Ivan Rupnik, L'arte della vita. Il quotidiano nella bellezza (Roma: Lipa, 2011), 234-35.

${ }^{38}$ David Maria Turoldo, Il fuoco di Elia profeta (Cinisello Balsamo: San Paolo, 2016), 91.

39 Jean Corbon, Liturgia alla sorgente, trad. il monastero Uspenskij di Roma (Magnano: Edizioni Qiqajon, 2003), 219.

${ }^{40}$ Corbon, Liturgia alla sorgente, 127.

${ }^{41}$ Cf. Daniélou, Platonisme et théologie mystique, 28.
} 
"bacio mistico", ha per scopo di mettere l'anima in contatto con il Verbo incarnato, dal quale solo vengono la luce e la grazia ${ }^{42}$.

In questo clima, la liturgia forma il modo di essere cristiano, lo "specifico cristiano", che può essere individuato su tre atteggiamenti secondo Grasso: accogliere la salvezza e comprenderla come un "dono": "vissuta come dono perché accettata pure attraverso un dono»; cogliere una presenza "cristica" densissima «per cui realtà della terra diventano per lui, e lo sono realmente nella loro essenza tutta nuova, Gesù Cristo»; il cristiano confessa il proprio peccato e chiede come dono «una giustizia superiore a quella degli scribi e dei farisei» ${ }^{43}$.

\section{LA CELEBRAZIONE LUOGO DELLA CONTEMPLAZIONE}

La Costituzione conciliare sulla liturgia ha stabilito, come uno degli scopi della riforma liturgica, che i fedeli possano partecipare alle celebrazioni liturgiche «consapevolmente, attivamente e fruttuosamente» (SC 11); e aggiunge: «partecipino all'azione sacra consapevolmente, piamente e attivamente, siano istruiti nella Parola di Dio, si nutrano alla mensa del corpo del Signore, rendano grazie a Dio, offrendo l'ostia immacolata [...] imparino ad offrire se stessi» (SC 48). Bisogna chiarire subito che la questione della partecipazione attiva non si esaurisce in un insieme di "azioni" esteriori, che rischiano di degenerare la liturgia in un certo "agire comune". Si tratta, infatti, di «introduzione in quell'actio essenziale che costituisce la liturgia, nella potenza trasformatrice di Dio, che attraverso l'evento liturgico vuole trasformare noi stessi ed il mondo» ${ }^{4}$.

Una celebrazione liturgica viva e genuina è il luogo privilegiato della contemplazione. Nella celebrazione «sta il culmine, il contatto autentico col Signore e con la sua opera salvifica, la vetta della realtà; qui soprattutto il Signore è presente in mezzo alla Chiesa che celebra le azioni liturgiche (SC 7)» ${ }^{45}$. La celebrazione «è un momento fontale, in cui il fiume di vita rinno-

${ }^{42}$ Cf. Daniélou, Platonisme et théologie mystique, 34.

${ }^{43}$ Giacomo Grasso, "Liturgia e spiritualità. Riflessione teologica," in Liturgia e spiritualità, ed. Secondo Mazzarello (Torino: Marietti, 1981), 42-43.

${ }^{44}$ Joseph Ratzinger, "Lo spirito della liturgia," in Teologia della liturgia, ed. Edmondo Caruana e Pierluca Azzaro, trad. Ingrid Stampa (Città del Vaticano: Libreria Editrice Vaticana, 2010), 166.

${ }^{45}$ Burkhard Neunheuser, "Spiritualità liturgica," in Liturgia, ed. Domenico Sartore, Achille Maria Triacca e Carlo Cibien (Cinisello Balsamo: San Paolo, 2001), 1920. 
va, fa crescere e vivifica gli alberi di vita [...] Perché la celebrazione sia trasfigurazione del corpo di Cristo bisogna che tutto l'uomo, che è corpo, vi sia coinvolto» ${ }^{46}$. Ogni celebrazione liturgica è caratterizzata da cinque dimensioni che la percorrono in ogni momento ed in ogni parte, sebbene in alcuni passaggi risultino maggiormente marcate ${ }^{47}$. Vivere intensamente queste dimensioni trasforma ogni celebrazione liturgica in una celebrazione contemplativa e realizza pienamente la partecipazione attiva:

1) La dimensione anamnetica che permette alla celebrazione, in quanto "azione liturgica", di diventare "memoriale", ossia "ripresentazione" efficace del Mistero di salvezza di Cristo.

2) La dimensione epicletica che rende efficace il memoriale, in virtù della presenza dello Spirito Santo essendo soggetto dell'anamnesi stessa.

3) La dimensione dossologica che attraversa tutta la celebrazione e si manifesta nel culto, nella lode, nell'adorazione, nella benedizione ascendente dell'uomo nei confronti di Dio.

4) La dimensione mistagogica che è la capacità della liturgia di aprire la mente e il cuore ai misteri che vengono celebrati. Essa introduce alla conoscenza e all'incontro con la salvezza celebrata.

5) La dimensione escatologica proietta la celebrazione verso il futuro e la rende un'anticipazione di quanto vivremo nel compimento della Storia della Salvezza.

Solo il simbolo in azione fa accedere al Mistero, e tutta l'azione contemplativa nella liturgia consiste nell'accedere al mistero attraverso i simboli che rendono i celebranti "partecipanti attivi" nella liturgia: «Un simbolo sorge quando qualcosa d'interiore, di spirituale, trova la sua espressione nell'esteriore» ${ }^{48}$. Infatti, è fondamentale in qualsiasi celebrazione liturgica vivere pienamente i momenti fondamentali della celebrazione come festa, come stare insieme, come preghiera comune, come silenzio e ascolto, come gratitudine e perdono. San Benedetto affermava: «mens nostra concordet voci nostrae»; da questo principio risulta che l'orante, nella liturgia, si lascia modellare dalle preghiere: «Queste ultime sono una finestra aperta sul mistero e sull'oggetto da contemplare e rivivere» ${ }^{49}$.

\footnotetext{
${ }^{46}$ Corbon, Liturgia alla sorgente, 121. 27.

${ }^{47}$ Cf. Muroni, Il Mistero di Cristo nel tempo e nello spazio, 39-52.

${ }^{48}$ Guardini, Lo spirito della liturgia, 64.

49 Magrassi, "Spiritualità liturgica. Riflessione pastorale," 49.
} 
Il culto cristiano, essendo un culto «in spirito e verità» (Gv 4,23), un culto «spirituale» $(\mathrm{Rm} 12,1)$, esige altra cosa che l'emozione esterna di una sensibilità passeggera. Questa disposizione profonda, formata in modo teologale, l'eucologia latina la riconosce sotto il nome di affectus. Dal punto di vista dell'antropologia liturgica e della spiritualità liturgica, l'affectus fa coppia con intellectus. Il primo indica la sensibilità convertita ed evangelizzata, il secondo indica l'intelligenza convertita ed evangelizzata. Questo processo, che esige una dimensione contemplativa, fa entrare il soggetto liturgico in una com-passione reale con il Cristo e la totalità del suo mistero pasquale. In questo modo, la celebrazione liturgica sfocia in un'autentica vita in Cristo ${ }^{50}$. Il momento liturgico, per sua natura, «fa comprendere al credente la totalità del Santo e l'operazione santificatrice del Santo di Dio, quel Cristo che lo ha salvato» ${ }^{51}$.

Secondo l'analisi di Špidlík, per i Padri, soprattutto i Padri del Vicino Oriente, «era del tutto naturale per loro collegare l'esperienza della contemplazione più alta con la celebrazione liturgica della comunità ecclesiale» ${ }^{52}$. Quest'aspetto si rivela come una conseguenza normale quando riprendiamo coscienza che «tutto nell'azione liturgica dice la grandezza di Dio e la sua gloria» ${ }^{53}$. Per questo motivo possiamo parlare del «primato dell'ascolto nel dialogo, della ammirazione e della lode nella "contemplazione" [...] E dal silenzio attonito dell' accoglienza della Parola sgorga la lode che si unisce al canto senza fine della beata Gerusalemme» ${ }^{54}$.

Secondo Efrem il Siro, la fede si nutre e cresce dalla lode, che a sua volta cresce a causa della meraviglia. La lode divina, che costituisce l'essenza della liturgia, compie lo scopo per il quale siamo stati creati; la lode distingue l'uomo vivente dagli altri esseri. Per Efrem, la lode comincia ad esprimersi nella sua dimensione "vocale" ma «quanto più raffinata e purificata diviene, tanto più assume il carattere della lode silenziosa degli esseri angelici» ${ }^{55}$. Per Efrem, si passa dalla lode vocale alla lode silenziosa, o per meglio dire lode "contemplativa". Per il nostro autore, questo movimento corrisponde al movimento di Dio che «passa dal Silenzio del suo Essere ineffabile alla Paro-

${ }^{50}$ Cf. François Cassingena-Trévedy, Les Pères de l'Église et la liturgie (Paris: Desclée de Brouwer, 2009), 293-94.

${ }^{51}$ Grasso, "Liturgia e spiritualità. Riflessione teologica," 43.

52 Špidlík e Gargano, La spiritualità dei Padri greci e orientali, 171.

53 Grasso, "Liturgia e spiritualità. Riflessione teologica," 43.

${ }^{54}$ Magrassi, "Spiritualità liturgica. Riflessione pastorale," 57-58.

${ }^{55}$ Sebastian P. Brock, L'occhio luminoso. La visione spirituale di sant'Efrem, trad. Maria Campatelli (Roma: Lipa, 1999), 87. 
la divina, il Verbo» ${ }^{56}$. Nell'Eucaristia si entra in questa condizione, in questo processo di nuova creazione. Andando nella stessa linea di pensiero, Grammont considera che la liturgia accompagna il credente alla soglia dove il silenzio e la contemplazione prendono il posto della parola, dove l'immobilità davanti al mistero di Dio diventano la lode silenziosa dell'adorazione ${ }^{57}$.

Nella sua teologia mistica-sacramentale, Dionigi l'Areopagita ritiene che ciò che il Cristo è, ciò che compie in nostro favore e la comunione mistica con Lui, si manifesta solo sotto la forma sacramentale. Solo nella partecipazione alla presenza effettiva di Cristo sull'altare siamo messi in comunicazione mistica con Lui. Da qui risulta che nella liturgia della comunità radunata abbiamo un'autentica esperienza di Cristo. Con questa considerazione, Dionigi raggiunge Filosseno di Mabbug ${ }^{58}$; per entrambi la conoscenza di Dio trascende l'investigazione razionale. Filosseno parla della "meraviglia", eredità di Efrem, come via della conoscenza mistica. Dionigi indica la strada della "non conoscenza" apofatica, dove i Nomi di Dio sono oggetti di lode. I due atteggiamenti, "meraviglia" e "non conoscenza", sono strettamente legati al vissuto liturgico. Questa fu la caratteristica della mistica siro-ortodossa, concepita piuttosto sotto la forma della mistagogia ${ }^{59}$.

In ogni celebrazione liturgica si risvegliano la nostalgia di un compimento escatologico e la contemplazione del "Sole invisibile". Questo è il traguardo di ogni contemplazione liturgica e di ogni celebrazione misterica. Negli ambienti monastici della Tebaide, per esempio, quest'attesa si traduceva liturgicamente nelle „vigilie”. L'attesa del sole visibile era il simbolo dell'attesa del sorgere del "Sole invisibile", del "Sole di giustizia" che illumina con i suoi raggi il cuore del contemplativo in attesa di trasformarlo nella sua realtà gloriosa ${ }^{60}$.

${ }^{56}$ Brock, L'occhio luminoso, 87.

${ }^{57}$ Grammont, "Liturgie et contemplation," 120.

${ }^{58}$ Filosseno, vescovo di Mabbug (nel nord dell'attuale Siria), fu uno dei più grandi teologi di questo tempo. Morì nel 523. Fu una figura guida nell'opposizione siro-ortodossa al Concilio di Calcedonia. Sebbene la maggior parte delle sue opere abbia un carattere prevalentemente teologico, trovò tempo anche per scrivere una serie di importanti opere nel campo della spiritualità. Filosseno offre una fusione originale d'eccezione della tradizione greca e di quella siriaca.

${ }^{59}$ Cf. Emiliano Fiori, "Mystique et liturgie. Entre Denys l'Aréopagite e le livre de Hiérothée: aux origines de la mystagogie syro-occidentale," in Les mystiques syriaques, ed. Alain Desreumaux (Paris: Geuthner, 2011), 39-40.

${ }^{60}$ Cf. Daniélou, "Le symbolisme des rites baptismaux," 25. 
Per terminare questa nostra riflessione, possiamo affermare che la liturgia è il luogo per eccellenza per la vita mistica e contemplativa. L'orante è situato nel pieno del Mistero, laddove attraverso le cose di questo mondo traspare, agli occhi della fede, il mondo nuovo, il mondo della risurrezione di Cristo, dove lo Spirito trasfigura incessantemente la faccia della terra ${ }^{61}$. La liturgia educa il nostro sguardo e lo purifica, in modo che nella fede, nella carità e nella speranza santa, incontra lo sguardo di Dio e si lascia invadere dalla sua presenza. La presenza trasfigurante di Dio "divinizza" come piace dire agli Orientali ${ }^{62}$. La liturgia è certamente ordinata a tale contemplazione «come alla propria perfezione interiore, non già per cessare di essere liturgia, ma per diventare liturgia contemplativa ${ }^{63}$. Da una vera liturgia contemplativa «la vita cambia gusto, assume un senso. È vissuta in riferimento più o meno costante a questo Dio. Emergono luci nuove sulla mia vita, alla luce del piano di Dio, si precisa il significato del mio essere in Dio e in questo mondo assieme ai fratelli» ${ }^{64}$. In questo modo, ogni credente «impegnato a testimoniare il Signore Gesù e ad annunziare il buon annunzio di salvezza, trova nella liturgia, momento denso e ultimo della sua esperienza cristiana, quanto deve avere per rendere densa e piena di sale la sua testimonianza» ${ }^{65}$.

Guardini, con pertinenza, mette in risalto la necessità della dimensione contemplativa della liturgia, in rapporto con il Lógos divino, Parola viva. Questa Parola, destinata a fruttificare nel cuore dell'orante, cerca un'accoglienza nel silenzio e nella calma contemplativa per dare tempo al seme di morire e di crescere:

Il tono di fondo della vita genuina e sana è contemplativo. L'energia della volontà, dell'azione della ricerca, per quanto intensa possa diventare, deve riposare sopra una profondità che è calma, che s'affissa nelle immutabili verità eterne. Questo è il sentire che ha le sue radici nell'eternità. Esso ha la pace; possiede quella serenità immune da tensioni che rappresenta la vittoria sopra la vita. Non ha fretta, ha tempo: può pertanto attendere e lasciar crescere [...] Nella liturgia il Lógos ha la preminenza, che gli spetta, sulla volontà. Di qui la sua mirabile

${ }^{61}$ Joseph Gelineau, “Approches doctrinales," in Liturgie et vie spirituelle (Paris: Éditions Beauchesne, 1977), 116.

${ }^{62}$ Grammont, "Liturgie et contemplation," 127.

${ }^{63}$ Vagaggini, Il senso teologico della liturgia, 690.

${ }^{64}$ Giuseppe Sovernigo, "Personalità ed esperienza di Dio nella liturgia. Aspetti psicologici,"in Liturgia Soglia dell'esperienza di Dio?, ed. Aldo Natale Terrin (Padova: Edizioni Messaggero, 1982), 190.

${ }^{65}$ Grasso, "Liturgia e spiritualità. Riflessione teologica," 44. 
placidità, la sua calma profonda. Di qui s'intende com'essa sembri totalmente risolversi in contemplazione, adorazione, esaltazione della verità divina ${ }^{66}$.

\section{BIBLIOGRAFIA}

Augé, Matias. Spiritualità liturgica. «Offrite i vostri corpi come sacrificio vivente, santo e gradito a Dio». Cinisello Balsamo: San Paolo, 1998.

Brock, Sebastian P. L'occhio luminoso. La visione spirituale di sant'Efrem. Tradotto da Maria Campatelli. Roma: Lipa, 1999.

Cassingena-Trévedy, François. Les Pères de l'Église et la liturgie. Paris: Desclée de Brouwer, 2009.

Castellano, Jesús. Liturgia y vida espiritual. Teología, celebración, experiencia. Barcelona: Centre de Pastoral Litúrgica, 2006.

Corbon, Jean. Liturgia alla sorgente. Tradotto dal monastero Uspenskij di Roma. Magnano: Edizioni Qiqajon, 2003.

Daniélou, Jean. Platonisme et théologie mystique. Essai sur la doctrine spirituelle de saint Grégoire de Nysse. Paris: Éditions Montaigne, 1944.

Daniélou, Jean. "Le symbolisme des rites baptismaux." Dieu Vivant 1(1945): 17-43.

Duggan, Robert. "Liturgical spirituality and liturgical reform." Spiritual Life 27(Spring 1981), 1: $46-53$.

Fernandez Rodriguez, Pedro. “Contemplación y Liturgia.” Ciencia Tomista 95(Julio-Septiembre 1968), 304: 483-505.

Fiori, Emiliano. "Mystique et liturgie. Entre Denys l'Aréopagite e le livre de Hiérothée: aux origines de la mystagogie syro-occidentale." In Les mystiques syriaques, édité par Alain Desreumaux, 27-44. Paris: Geuthner, 2011.

Gregorio di Nissa, Omelie sul Cantico dei cantici. Tradotto da Claudio Moreschini. Roma: Città Nuova Editrice, 1988.

Grelot, Pierre, Emmanuel Lanne, Pierre-Marie Gy, Niels Krogh Rassumen, Boris Bobrinskoy, Joseph Gelineau, Paul Grammont, et Irénée-H. Dalmais. Liturgie et vie spirituelle. Paris: Éditions Beauchesne, 1977.

Grillo, Andrea. Eucaristia. Azione rituale, forme storiche, essenza sistematica. Brescia: Queriniana, 2019.

Guardini, Romano. Lo spirito della liturgia. I santi segni. Tradotto da Mario Bendiscioli. Brescia: Morcelliana, $2007^{11}$.

Maritain, Jacques, et Raïssa Maritain. Liturgie et contemplation. Bruges: Desclée de Brouwer, 1959.

Muroni, Pietro Angelo. Il Mistero di Cristo nel tempo e nello spazio. La celebrazione cristiana. Roma: Urbaniana University Press, 2014.

Neunheuser, Burkhard. "Spiritualità liturgica". In Liturgia, editto da Domenico Sartore, Achille Maria Triacca, e Carlo Cibien, 1915-36. Cinisello Balsamo: San Paolo, 2001.

Origene, La preghiera. Tradotto da Giuseppe Del Ton. Roma: Arnoldo Mondadori Editore, 1984.

${ }^{66}$ Guardini, Lo spirito della liturgia, 109-10. 
Pacomio, Luciano, Giacomo Grasso, Mariano Magrassi, e Secondo Mazzarello. Liturgia e spiritualità. Torino: Marietti, 1981.

Paolo VI. "Omelia nella $9^{\text {a }}$ sessione pubblica. 7 dicembre 1965." Enchiridion Vaticanum 1(1962-1965): 292-311.

Pseudo-Clemente. "Omelia dello Pseudo-Clemente". In I Padri apostolici, editto e tradotto da Antonio Quacquarelli, 215-34. Roma: Città Nuova Editrice, $1981^{3}$.

Ratzinger, Joseph. "Lo spirito della liturgia". In Teologia della liturgia, editto da Edmondo Caruana, e Pierluca Azzaro, 25-217. Tradotto da Ingrid Stampa. Città del Vaticano: Libreria Editrice Vaticana, 2010.

Régamey, Pie-Raymond. "L'orientation contemplative de la prière liturgique." La Vie Spirituelle 102 (Mai 1960): 461, 469-93.

Rosso, Stefano. Un popolo di sacerdoti. Introduzione alla liturgia. Leumann: Elledici, $2007^{2}$. Rupnik, Marco Ivan. L'arte della vita. Il quotidiano nella bellezza. Roma: Lipa, 2011.

Schmemann, Alexander. Per la vita del mondo. Il mondo come sacramento. Tradotto da Maria Campatelli. Roma: Lipa, 2012.

Špidlík, Tomáš, e Innocenzo Gargano. La spiritualità dei Padri greci e orientali. Roma: Borla, 1983.

Terrin, Aldo Natale, ed. Liturgia Soglia dell'esperienza di Dio? Padova: Edizioni Messaggero, 1982.

Turoldo, David Maria. Il fuoco di Elia profeta. Cinisello Balsamo: San Paolo, 2016.

Vagaggini, Cipriano. "Contemplazione nella Liturgia e Contemplazione fuori della Liturgia." Rivista di ascetica e mistica 7(Gennaio-Febbraio 1962), 1: 8-34.

Vagaggini, Cipriano. Il senso teologico della liturgia. Roma: Edizioni Paoline, $1965^{4}$.

\title{
LA DIMENSIONE CONTEMPLATIVA DELLA LITURGIA
}

\author{
$\mathrm{S}$ o $\mathrm{m} \mathrm{m}$ a r i o
}

Il rapporto tra liturgia e contemplazione è basilare per il vissuto cristiano. La contemplazione, per sua natura, è "misterica", perché ha come oggetto i misteri salvifici, proclamati dalla Parola, compiuti pienamente nella persona di Cristo, celebrati dalla liturgia. Ogni contemplazione autentica deve nutrirsi della Parola proclamata e della celebrazione dei misteri della Salvezza. D'altronde, ogni liturgia è ordinata alla contemplazione come alla propria perfezione, non per cessare di essere liturgia, ma per diventare liturgia contemplativa. Il nostro articolo cerca di mettere in luce il rapporto inscindibile tra questi due aspetti che formano il "modo di essere cristiano". Questo rapporto garantisce, allo stesso tempo, l'autenticità della contemplazione e la fruttuosità della partecipazione alla celebrazione liturgica.

Parole chiavi: liturgia; contemplazione; battesimo; mistero; celebrazione; Chiesa. 


\section{KONTEMPLACYJNY WYMIAR LITURGII}

\section{S t r e s z c z e n i e}

Relacja między liturgią a kontemplacją jest fundamentalna dla życia chrześcijańskiego. Kontemplacja ze swej natury jest „tajemnicą”, ponieważ ma za przedmiot głoszone przez Słowo tajemnice zbawcze, które wypełniają się w pełni w Osobie Chrystusa, sprawowane w liturgii. Każda autentyczna kontemplacja powinna być karmiona głoszonym Słowem i celebracją tajemnic zbawienia. Z drugiej strony, każda liturgia jest skierowana na kontemplację własnej doskonałości tak, aby nie przestała być liturgią, ale stała się liturgią kontemplacyjną. Artykuł stara się podkreślić nierozerwalny związek między tymi dwoma aspektami, które składają się na „sposób bycia chrześcijaninem”. Relacja ta gwarantuje jednocześnie autentyczność kontemplacji i owocność uczestnictwa w celebracji liturgicznej.

Słowa kluczowe: liturgia; kontemplacja; chrzest; tajemnica; celebracja; Kościół. 